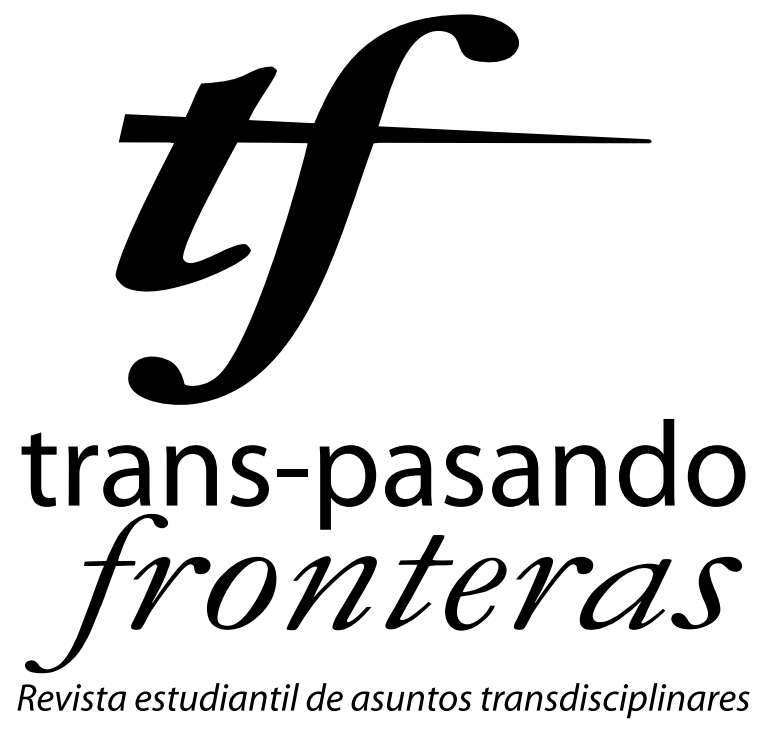

Una publicación de

Estudios

Interdisciplinarios

Jurídicos, Sociales

000 y Humanistas

ICESS

FACULTAD DE

DERECHO Y CIENCIAS

SOCIALES

然 UNIVERSIDAD 


\title{
EI Sistema interamericano de defensa como garante de la seguridad regional
}

\author{
Sebastián Acosta Zapata \\ (saz0913@hotmail.com)
}

GALVÉZ, Arturo (2001). Sistema Interamericano de Defensa. Barranquilla : Editorial Universidad de Norte, 203 pp.

"El factor tiempo es de vital consideración y vital es la correcta interpretación del significado del término para nuestro programa de defensa nacional" George Marshall

El libro Sistema Interamericano de Defensa es un recuento sustancioso del abogado barranquillero Arturo Gálvez, quien se ha desempeñado como profesor universitario de Relaciones Internacionales (RR.II.), asesor y representante de la Cancillería colombiana en temáticas como las fronteras marítimas, el derecho del mar y la soberanía territorial. Él hace una recopilación de todos los intentos $\mathrm{y}$ las gestiones de tratados que desembocan en normas jurídico-políticas internacionales. Los Estados Americanos han he- cho importantes avances desde sus inicios republicanos post-independentistas para garantizar la seguridad estatal y regional de América frente a intervenciones estatales extranjeras.

El eje principal del libro es tratar las Relaciones Internacionales como sistemas multilaterales para la consecución de objetivos en común, la configuración de instituciones regionales, la construcción y el mantenimiento de la paz en un siglo tan convulsionado como el XX, teniendo en cuenta sus inicios en el siglo XIX. Además, muestra la importancia de los regímenes democráticos en el cuadro de las relaciones internacionales para el sostenimiento de la paz en la historia reciente del continente americano. 
El desarrollo de hechos importantes (como coyunturas) para el establecimiento de marcos jurídicos y comportamientos políticos, en el plano interamericano, es esencial para tomar un hecho empírico regional que se preocupa por la seguridad, el cual puede tener un referente teórico combinado: la teoría realista y la paz democrática -que son usadas para relacionar y analizar lo empírico y lo teórico.

Situaciones particulares han planteado retos para la aplicabilidad de los diferentes tratados que configuran el Sistema Interamericano de Defensa y el Tratado Interamericano de Asistencia Recíproca (T.I.A.R.). Como la Guerra de las Malvinas (1982) y las diferencias propias de los intereses estatales en temas particulares como la seguridad económica, la no intervención militar y la defensa de la democracia.

\section{Argumentos principales}

El texto muestra el proceso de formación del Derecho Internacional Americano bajo la organización de distintos congresos, reuniones y conferencias. Éstas han aportado principios y elementos para organizar el escenario interamericano, tendiendo como tema principal lo relativo a la defensa del continente.

El primer intento se remonta al periodo post-independencia de América Latina en el que el ideario del libertador Simón Bolívar, contribuye a la elaboración de un orden jurídico que es la base del Tratado de Unión, Liga y Confederación Perpetua de 1826, del cual evoluciona el principio de la solidaridad defensiva americana.

Los Estados americanos en procura de acuerdos comunes, lo único que encontraron fue un "hispanoamericanismo" débil que impidió el desarrollo de los ideales bolivarianos. Esto generó un vació que dio cabida al surgimiento del "panamericanismo" producto de la Doctrina Monroe de 1823 que abarca de 1888 a 1948, año en que se crea la Organización de Estados Americanos (OEA). A pesar de esta desviación del sistema defensivo, los principios básicos son: 1) la no aceptación de la legitimidad del título de la conquista; 2) la no intervención; y 3) la igualdad jurídica de los Estados americanos, no fueron abandonados por sistema interamericano.

Además, surgió la consulta como instrumento jurídico para estudiar todas las amenazas o actos de agresión que se puedan perpetrar contra las repúblicas americanas. Este instrumento es el resultante de 
una necesidad frente al peligro bélico. La consulta incorpora su valor en el Acta de Chapultepec de 1945 en la cual se formaliza una exigencia histórica que procura por la construcción de un tratado que recogiera los principios básicos de la defensa colectiva con el fin de generar unidad y defensa.

El Sistema interamericano de defensa es reconocida en la Carta de las Naciones Unidas por la disposición del derecho inmanente de legítima defensa individual y colectiva. Esto permitió estructurar el T.I.A.R. de 1947 hecho en Río de Janeiro. Allí se establece el principio de la "solidaridad defensiva americana ante la agresión" y cuando las repúblicas americanas lo aplican, instan el derecho a la legítima defensa individual o colectiva.

Este Tratado firmado en Río de Janeiro se articuló con lo propuesto por la Organización de las Naciones Unidas (ONU) y la OEA, sin embargo tuvo que reformarse dada la crisis producto del régimen de Castro en Cuba, de las intervenciones de Estados Unidos y por las aplicaciones politizadas del Tratado. Los objetivos principales de la reforma de 1975 fueron hacer un instrumento más acorde con las realidades históricas, la libre autodeterminación y la seguridad económica colectiva.

A pesar de lo anterior, por un lado, las disposiciones del T.I.A.R. están sometidas a cargas políticas, su interpretación es relativa y ha generado falta de certeza sobre su eficacia. Y por el otro lado, la aplicación del Tratado en cuestión se ha centrado en conflictos entre los Estados americanos y el Órgano de Consulta que dedica sus acciones al restablecimiento inmediato de las relaciones entre las partes en conflicto a su estado anterior al ataque armado.

Por lo tanto, "la aplicación de medidas coercitivas por un acuerdo regional como el Tratado de Río de janeiro no garantiza siempre la normalización de las relaciones interamericanas sino que, por el contrario, puede incrementar reacciones adversas a la paz y a la seguridad internacional" (Gálvez, 201). Mucho de esto tiene que ver con que las acciones son más de carácter político que jurídico, dejando de lado el derecho y centrándose en la ideología política del momento.

\section{Sustento para el Análisis Teórico de hechos Empíricos}

Teniendo en cuenta referencias teóricas y los análisis pertinentes que se le deben hacer al libro, se propone una perspectiva a la 
luz de Hans Morgenthau ${ }^{1}$ y su teoría realista de las RR.II., ya que es claro que el actor principal en este recorrido histórico del Sistema interamericano de defensa son los Estados-nación. Pistas para corroborar la anterior afirmación se encuentran, primero, en el hecho que todo inicie cuando las naciones hispanoamericanas se independizan y ven la necesidad de preservar su interés en términos de Poder y de consolidar el orden administrativo de los Estados independientes. Segundo, en que la defensa, el poder en términos de fuerza militar y la seguridad son aspectos importantes para la teoría realista $\mathrm{y}$ esto se evidencia fuertemente en el interés de los Estados para cumplir sus metas políticas contextuales en cuanto a seguridad estatal en un marco regional.

Otra importante propuesta teórica para explicar la conformación del Sistema interamericano de defensa es la denominada "Paz democrática" del trabajo de Zeno Gobetti ${ }^{2}$, porque la preocupación que se plantean por garantizar la paz entre los Estados americanos entre sí y con el resto del

1 MORGENTHAU, Hans J. (1986). Politica entre las naciones: la lucha por el poder y la paz [título original: Politics Among Nations: The Struggle for Power and Peace]. Grupo Editor Latinoamericano - GEL.

2 GOBETTI, Zeno (2009). "Una revisión de la teoría de la paz democrática”. En: Revista CS, No. 3 "Paz y seguridad humana", pp. 39-74. Cali: Facultad de Derecho y Ciencias sociales, Universidad Icesi. mundo se centra, de forma concluyente y explícita, en el mantenimiento de regímenes democráticos en todo el continente.
"Podemos identificar dos presupues- tos fundamentales. En primer lugar, la teoría de la paz democrática se basa en la idea de que es posible limitar algu- nos aspectos negativos de la situación de anarquía del sistema internacional [...]. En segundo lugar, esta teoría pierde su sentido si no se reconoce el hecho de que los Estados tienen dife- rentes comportamientos en el sistema internacional y que el régimen politi- co influye de manera sustancial en tal comportamiento” (Gálvez, 1)

En este párrafo están muy bien condensadas las ideas que están presentes en la configuración del Sistema interamericano de defensa en relación con el mantenimiento de las democracias como reza la resolución XXII sobre "Preservación y Defensa de la Democracia en América Latina" planteada para el mantenimiento de una clase de sistema de defensa político en Bogotá en 1948.

\section{Reflexiones finales}

Finalmente el texto plantea, en primer término, que el grado de tensión política existente siempre influirá sobre las interpretaciones regionalistas o univer- 
salistas que se les den a Tratados Regionales. En segundo término, que los hechos como la Guerra de las Malvinas de 1982 y recientemente recordada, la inestabilidad política de Centroamérica dado el problema del narcotráfico, la percepción de la pérdida de poder político, mas no militar de Estados Unidos, definirá la estrategia futura de la solidaridad defensiva del continente en cuanto al ámbito político como jurídico. Y en tercer lugar, que el deseo de las repúblicas americanas por garantizar la defensa de las democracias representativas como sistema político coincide en el uso de los diferentes principios y tratados convenidos que procuran la aplicación de mecanismos apropiados, pero teniendo en cuenta la naturaleza del Tratado de Legítima Defensa Interamericano.

El texto es pertinente para construir una idea sólida sobre la conformación del Sistemas internacionales de defensa. Hace un barrido por los principales hechos en el plano americano, que centran en la defensa y en el desarrollo de planes y estrategias, los garantes de la defensa y la paz en la región los cuales se ven representados en los diferentes Tratados y en la formalidad de las relaciones internacionales de los países del continente americano.
Muestra con objetividad las limitaciones del derecho en comparación con los alcances de la política y de la jerarquía interamericana. Coincido con el autor que los tratados no son cartas de absoluta aplicación a las relaciones políticas a nivel internacional, de lo cual surgen preguntas sobre el episodio de Malvinas y la posición lejana que tomó Estados Unidos en plena guerra fría en relación a Reino Unido, habiendo pasado este mundo bipolar, ¿Estados Unidos actuará igual que hace 30 años? Analizando también la relación del sistema de defensa de la OEA y la fuerte influencia de EE.UU. en la creación de este organismo y su alcance en América Latina, genera preguntas por el grado de determinación de los Estados Unidos en la OEA o que dada la situación actual de la potencia americana, los demás países serían más autónomos en las decisiones o prevalecería la fuerza militar a la fuerza política norteamericana

En relación a cuestiones más reales y actuales surgen también inquietudes como ¿cuál es la fuerza y alcance que el Sistema interamericano de defensa tiene frente al "Crimen Organizado Transnacional" que es una de las principales amenazas en la mayoría de los Estados americanos actualmente? 
Es claro que tanto el texto como el Sistema interamericano de defensa se preocupan por la amenaza estatal extranjera a América. Y si se quiere profundizar en este tema tomando a los Estados como máximos actores del sistema internacional. El libro es muy enriquecedor y da a conocer el desarrollo de tratados para garantizar la Defensa y la Democracia de América. 